INPLASY

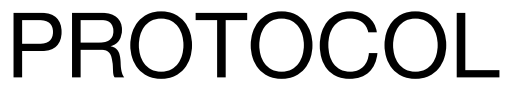

To cite: Young et al. A Scoping Review of interpreter-mediated assessments under the Mental Health Act (1983) and international equivalents. Inplasy protocol 202220086. doi:

10.37766/inplasy2022.2.0086

Received: 20 February 2022

Published: 21 February 2022

Corresponding author:

Alys Young

alys.young@manchester.co.uk

Author Affiliation:

University of Manchester

Support: NIHR SSCR (Ref \#P172).

Review Stage at time of this submission: Formal screening of search results against eligibility criteria.

Conflicts of interest:

None declared.

\section{A Scoping Review of interpreter- mediated assessments under the Mental Health Act (1983) and international equivalents}

Young, A; Rodríguez Vicente, N2; Tipton, R33 Napier, J4; Vicary, S5; Hulme, C6.

Review question / Objective: The purpose of this scoping review is to identify and evaluate available evidence concerning assessments under the Mental Health Act (1983) (MHA) (and international equivalents) which are carried out with the assistance of a spoken or signed language interpreter. 'International equivalents' refers to pieces of legislation in countries other than England and Wales that concern formal assessment for compulsory assessment and treatment, including hospital detention, with respect to a mental disorder. [Both the specific Act that applies to England and Wales and its international equivalents are henceforth referred to as MHA]. The guiding questions are: - What are the enablers and barriers to good practice in interpreter mediated MHA assessments? - To what extent and how might interpreter mediation support or impede the legal rights and best interests of those assessed under the MHA? The aim is to determine whether the body of research available to date is sufficient to inform evidence-based guidelines for interpreters and for mental health professionals, in particular those who have the duty to make decisions under the MHA, known in England and Wales as Approved Mental Health Professional (AMHPs), to work in a joint and effective manner.

INPLASY registration number: This protocol was registered with the International Platform of Registered Systematic Review and Meta-Analysis Protocols (INPLASY) on 21 February 2022 and was last updated on 21 February 2022 (registration number INPLASY202220086).

\section{INTRODUCTION}

Review question / Objective: The purpose of this scoping review is to identify and evaluate available evidence concerning assessments under the Mental Health Act (1983) (MHA) (and international equivalents) which are carried out with the assistance 
of a spoken or signed language interpreter. 'International equivalents' refers to pieces of legislation in countries other than England and Wales that concern formal assessment for compulsory assessment and treatment, including hospital detention, with respect to a mental disorder. [Both the specific Act that applies to England and Wales and its international equivalents are henceforth referred to as MHA]. The guiding questions are: - What are the enablers and barriers to good practice in interpreter mediated MHA assessments? To what extent and how might interpreter mediation support or impede the legal rights and best interests of those assessed under the MHA? The aim is to determine whether the body of research available to date is sufficient to inform evidence-based guidelines for interpreters and for mental health professionals, in particular those who have the duty to make decisions under the MHA, known in England and Wales as Approved Mental Health Professional (AMHPs), to work in a joint and effective manner.

Rationale: Creating the best communication possible or, as the Code of Practice to states, "interviewing in a suitable manner" (Department of Health 2015 para 14.49) is a priority during MHA assessments. In these events, there is a requirement to ensure a person's language needs are fully met if they do not use spoken English. This language gap can be bridged through spoken language and sign language interpreters. There has been growing attention to differences in outcomes of MHA assessments involving members of some Black, Asian and Minority Ethnic (BAME) communities in England and Wales (DHSC 2018; DHSC, 2019). However, this is not the same as paying attention to best practice in cases where a person's language may differ from English and where an interpreter is required. (Not all members of minority ethnic communities use a language other than spoken English). This scoping review aims to address this gap in the literature.

Condition being studied: This scoping review concerns people assessed under the MHA through signed or spoken language interpreters. People assessed under the MHA may experience a range of mental disorders that might require them to be detained in hospital for assessment and/or treatment (or under treatment orders in the community) if they are unable or unwilling to be detained or receive treatment on a voluntary basis. Detention in hospital (and mandatory Community Treatment Orders) result if an individual is a danger to themselves or others without such an order/detention being in place.

\section{METHODS}

Search strategy: The methods for this review are informed by the nine-step scoping review framework (Peters et al., 2020) expanded from Arksey and O'Malley (2005) and Levac et al (2010) and will be reported following the PRISMA Extension for Scoping Reviews (PRISMA-ScR) (Tricco et al., 2018). Bibliographic databases (see below) and forward citation sources from reference lists of identified articles as well as grey literature sources (e.g., policy, practice, and guidelines documents) will be searched. Examples of key words include 'MHA assessments', 'adult mental health' 'compulsory detention' and 'mental health interpreters'. Techniques for conducting the systematic literature search will include (i) the use of free-text words, (ii) truncation (e.g. sign* language will generate the words: sign language and signed language), (iii) use of Boolean operators (e.g. AND, OR). The online systematic review management system COVIDENCE will be used to assist with the search and retrieval process.

Participant or population: Professionals involved in MHA assessments including AMHPs (in England and Wales), doctors and interpreters (signed and spoken languages) and those subject to MHA assessments.

Intervention: N/A.

Comparator: N/A. 
Study designs to be included: Quantitative, qualitative and mixed methods empirical studies that address the subject of interpreter-mediated MHA assessments and meet the inclusion criteria.

Eligibility criteria: Inclusion criteria: 1. Item focuses on aspects related to the Mental Health Act (1983) or its international equivalents. 2. Item includes information or data on the following professional roles: Approved Mental Health Professionals (AMHPs) conducting MHA assessments, interpreters/translators, cultural advocates, language concordant professionals (and their international equivalent professional designations).3. Date range for item publication: 1980 - 2022 inclusive. 4. Items published in any of the languages of the multilingual investigator team namely: English, Spanish, French, BSL (British Sign Language), ASL (American Sign Language), International Sign, Dutch or German.5. Item concerns some aspects of interpreting within a formal mental health assessment, encompassing both signed and spoken language interpreting. 6. Item discusses interprofessional working dynamics between interpreters and other professionals (prioritising health and/or social care workers).7. Item is primary research including scholarly journal publications, book chapters, books and similar.8. Item is grey literature including reports, policy, legislation, professional guidance, regulatory/government reports, pre-prints, newsletters, and bulletins and similar.Exclusion criteria: 1. Item focuses primarily on discourse analytical or wider linguistic aspects of interpreting and translation with no direct link to issues around mental health assessment. 2. Item involves linguistically or culturally diverse populations with no need of communication support including interpreting. 3 . Item falls outside of publication date range (1980-2022 inclusive). 4. Item focuses on a mental health environment with no overlapping aims with/direct relevance to MHA assessments.

Information sources: Research data bases: PubMed - National Library of Medicine,
ASSIA: Applied Social Sciences Index and Abstracts, APA (American Psychological Association), Psyclnfo, Web of science (Clarivate), CINAHL (nursing/allied health), Social care online (SCIE), Scopus (Elsevier), EThOS - e-theses online service. Online indices of specific journals where publications on this topic were likely to be placed. For example: British Journal of Social Work, Health and Social Care in the Community, Translation and Interpreting Studies, Interpreting: Research \& Practice in Interpreting; Interpreting and Society; Communication \& Medicine. Subjectspecific publisher websites using the key terms listed in the search strategy in order to capture book chapters. Grey literature sources including profession-specific guidelines and governmental policy and legal statutes. Pre-existing professional and research knowledge from the authorship team who had professional experience as working AMHPs or predecessors Approved Social Workers (ASW) or as interpreters (spoken or signed languages) as well as cross-disciplinary academic knowledge.

Main outcome(s): Any report concerning mediated assessment through interpreters under the MHA will be included. The exploration and identification of challenges and good practice will result. A brief appraisal of the quality of evidence and evidence gaps will be summarised.

\section{Additional outcome(s): None.}

Data management: Study selection follows a two stage process: (i) title and abstract screening will be carried out by two people, one from a mental health social work background, one from an interpreting background and which results in a yes/no/ maybe conclusion applied to each item. Those designated 'maybe', or where there is a conflict of opinion between the two reviewers, will then be subjected to a discussion between reviewers to reach a consensus of yes or no; (ii) full text screening, by two reviewers from mental health social work and interpreting backgrounds resulting in a yes/no designation. A third reviewer will be used to 
resolve any conflicts. At both stages in the screening process the inclusion/exclusion criteria will be applied. Reasons for exclusion at either stage of study selection will be recorded. All items will be held in full text version within COVIDENCE. Relevant data from each selected study at stage (ii) screening will be extracted and charted using the COVIDENCE data charting tool. For studies generating primary data, descriptive data to be recorded will include year of publication, location, research design, methods, analytical approach, participant characteristics, setting, interventions (if any), comparison group (if any). Outcome data to be recorded will include results of quantitative and qualitative analyses, triangulated data (if any), comparative data analysis with other studies or official records (if any). For studies involving secondary data analysis, main outcome data and synthesis of findings will be recorded along with number of studies screened, approach to meta-analysis/ synthesis/ review, inclusion/ exclusion criteria. For items from grey literature including policy, guidelines and statutory instruments, descriptive data to be recorded will include details of the jurisdictions to which the item applies, the purpose/ intent of the document, the date and origin of its publication, contents considered relevant, specific data cited relevant to the review.

Quality assessment / Risk of bias analysis: A quality assessment review will be applied to items of primary data using the Crowe Critical Appraisal Tool (CCAT) which has been validated for quantitative and qualitative study designs (Crowe, Sheppard \& Campbell, 2011). No formal quality assessment tool will be used for items of secondary data analysis or grey literature.

Strategy of data synthesis: A narrative synthesis will be presented following a thematic structure generated by the evidence in the items reviewed and in line with the aims and objectives of the scoping review.
Subgroup analysis: As this is a scoping review, there is no plan for subgroup analysis.

Sensitivity analysis: As this is a scoping review, there is no plan for sensitivity analysis.

Language: The review includes publications in any of the languages of the multilingual investigator team: English, Spanish, French, BSL, ASL, International Sign, Dutch or German.

\section{Country(ies) involved: United Kingdom.}

References: Arksey H., O'Malley L. (2005) Scoping studies: towards a methodological framework, International Journal of Social Research Methodology, 8:1, 19-32, DOI: 10.1080/1364557032000119616;

DHSC (2015). Mental Health Act 1983: Code of Practice. https://www.gov.uk/ government/publications/code-of-practicemental-health-act-1983;

DHSC (2018). Modernising the Mental Health Act: Increasing choice, reducing compulsion. Final report of the Independent Review of the Mental Health Act 1983. https://www.gov.uk/government/ publications/modernising-the-mentalhealth-act-final-report-from-theindependent-review;

DHSC (2019). Independent Review of the Mental Health Act 1983: supporting d o c u m e n t s. h t t p s:// assets.publishing.service.gov.uk/ government/uploads/system/uploads/ attachment_data/file/778898/ Independent_Review_of_the_Mental_Health Act_1983_-_supporting_documents.pdf; Levac, D., Colquhoun, H. \& O'Brien, K.K. (2010). Scoping studies: advancing the methodology. Implementation Sci 5, 69 https://doi.org/10.1186/1748-5908-5-69; Peters MDJ, Marnie C, Tricco AC, Pollock $D$, Munn Z, Alexander L, Mclnerney $P$, Godfrey CM, Khalil H. (2020). Updated methodological guidance for the conduct of scoping reviews. JBI Evid Synth. 18(10):2119-2126. doi: 10.11124/ JBIES-20-00167;

Tricco AC, Lillie E, Zarin W, O'Brien KK, Colquhoun H, Levac D, Moher D, Peters 
MDJ, Horsley T, Weeks L, Hempel S, Akl EA, Chang C, McGowan J, Stewart L, Hartling L, Aldcroft A, Wilson MG, Garritty C, Lewin S, Godfrey CM, Macdonald MT, Langlois EV, Soares-Weiser K, Moriarty J, Clifford T, Tunçalp Ö, Straus SE. (2018). PRISMA Extension for Scoping Reviews (PRISMA-ScR): Checklist and Explana.

Keywords: Mental Health Act; Mental health interpreting; Sign language; Approved Mental health Professional (AMHP); Deprivation of liberty; interpreting and translation.

Dissemination plans: The scoping review findings will be published in peer-reviewed journals, presented at conferences, made available in summary form on the research project website (https://sites. manchester.ac.uk/informhaa/), of which this study is one component in plain English and BSL.

Contributions of each author:

Author 1 - Alys Young - AY developed the scoping review plan, acted as reviewer at stage (ii) screening, assisted with data extraction, led the narrative synthesis, cowrote the reporting of the results and assisted in manuscript preparation for publication.

Email: alys.young@manchester.co.uk

Author 2 - Natalia Rodríguez Vicente - NRV conducted a preliminary search of materials according to the inclusion criteria and uploaded such material onto COVIDENCE, led the charting of the data and quality appraisal in the data extraction phase, co-developed the narrative synthesis, and co-wrote the reporting of the results.

Email: natalia.rodriguezvicente@essex.ac.uk Author 3 - Rebecca Tipton - RT acted as reviewer at stage (ii) screening, codeveloped the narrative synthesis and cowrote the reporting of the results.

Email: rebecca.tipton@manchester.co.uk Author 4 - Jemina Napier - JN supported development of the scoping review plan, acted as reviewer at stage (i) screening, codeveloped the narrative synthesis, and cowrote the reporting of the results.

Email: j.napier@hw.co.uk
Author 5 - Sarah Vicary - SV supplied terminology for inclusion and exclusion criteria, suggested relevant databases, identified articles and assisted in manuscript preparation for publication.

Email: sarah.vicary@open.co.uk

Author 6 - Celia Hulme - $\mathrm{CH}$ assisted with the development of the scoping review plan and assisted in manuscript preparation for publication.

Email: celia.hulme@manchester.co.uk 